\title{
Malaria From Hyperendemicity to Elimination Along International Border in Yunnan, 2003-2020: a Case Study of Border Malaria Control and Elimination in China
}

Hui Liu

Yunnan Institute of Parasitic Diseases https://orcid.org/0000-0001-5227-3164

Yao-Wu Zhou

Yunnan Institute of Parasitic Diseases

Yan Deng

Yunnan Institute of Parasitic Diseases

Zu-Rui Lin

Yunnan Institute of Parasitic Diseases

Cang-Lin Zhang

Yunnan Institute of Parasitic Diseases

Qi-Yan Chen

Yunnan Institute of Parasitic Diseases

Chun Wei

Yunnan Institute of Parasitic Diseases

Kai-Xia Duan

Yunnan Institute of Parasitic Diseases

\section{Peng Tian}

Yunnan Institute of Parasitic Diseases

Hong-Ning Zhou ( $\nabla$ zhouhn66@163.com )

Yunnan Institute of Parasitic Diseases

Jian-wei Xu

Yunnan Institute of Parasitic Diseases

\section{Research Article}

Keywords: Case study, border malaria, control, elimination, international collaboration, Yunnan

Posted Date: November 16th, 2021

DOI: https://doi.org/10.21203/rs.3.rs-1069454/v1 
License: (c) (i) This work is licensed under a Creative Commons Attribution 4.0 International License. Read Full License 


\section{Abstract}

Background: Border malaria is always one of the most intractable problems in the path to malaria elimination worldwide. Movement of both human population and anophelines mosquitoes infected with Plasmodium spp can cause cross-border malaria transmission. The border areas of Yunnan were still malaria hyperendemicity in the early of this century.

Methods: This case study collected all available data related to border malaria surveillance and control in 25 border counties from 2003 to 2020 to analyses strategies, interventions and their efficacy.

Results: Number of malaria cases was a total of 10349 cases with an annual parasite incidence (API) 17.10 per 10000 person-years in the border area in 2003. From 2003 to 2013, integrated interventions based on natural village-based stratification, including mass drug administration for radical cure and preventive treatment, presumptive treatment of all febrile patients for malaria and indoor residual spraying or dipping bed nets with insecticides were successfully carried out. By 2013 , overall API was reduced to 0.62 per 10000 person-years, meanwhile, effective cross border collaboration for malaria control reduced malaria burden by $90 \%$ in neighbouring border areas of Myanmar. From 2017 with zero indigenous case forward, the comprehensive strategy, including universal coverage of surveillance to detect malaria cases, rapid response to any malaria cues and effective border collaboration with neighbouring successfully eliminated malaria and fully prevented reintroduction of malaria transmission in 25 border counties of Yunnan.

Conclusions: Border malaria elimination is an intensive intervention. The local context based strategy contributes to malaria control and elimination, and then prevent reintroduction of malaria transmission. Other border areas should do their own intervention trials to develop their own strategy.

\section{Background}

Globally, malaria is continuously one of public health problems [1]. According to the World Health Organization (WHO) World Malaria Reports 2019 and 2020, progress has slowed or even stalled. The morbidity slowed over the period 2016-2018 compared to the period 2010-2015. An estimated 228 million cases of malaria were reported worldwide in 2018 [1] and 229 million malaria cases reported in 2019 [2]. This trend is challenging the WHO's goal that achieve at least $90 \%$ global reduction of mortality rate and case incidence, malaria free at least 35 endemic countries by 2030 compared with $2015[3,4]$ and malaria elimination in all countries in the Greater Mekong Subregion (GMS) [5]. Yunnan with a population of 48 million shares $4060 \mathrm{~km}$ of border with Myanmar (1997 km), Laos (710 km) and Vietnam (1353 km). Yunnan's border areas have similar malaria ecology and vector system and social cultures with five other countries in the GMS, including diversity of landforms, climates, ethnicity, heavily forested environment, diversity in vector species and the tremendous spatial heterogeneity in distribution patterns. These factors imply original hyperendemicity in Yunnan's border area. Malaria along international border in Yunnan definitely affected elimination in China. Border malaria elimination would significantly 
contribute to China for the WHO malaria elimination certification. When malaria program is reorientating from control to elimination, border malaria is one of the most intractable problems in the path to malaria elimination. Frequent migrants and anophelines mosquitoes infected with Plasmodium spp crossing the border, and relatively underdeveloped health service can lead to cross-border transmission of malaria parasites [6, 7]. The objective of this case study was: 1) to analyses strategies and interventions used from malaria control to elimination and their impact during 2003-2020, and 2) to present strategy in preventing reintroduction of malaria transmission along the international border in Yunnan.

\section{Methods}

\section{Study site}

The border counties are defined as border areas in this study. There are 25 border counties in Yunnan, namely 17 counties bordering with Myanmar, one (Mengla) with Myanmar and Laos, one (Jiangcheng) with Laos and Vietnam, and six counties with Vietnam. A total of 909308 people (2020) were living in the border areas with a tropical or subtropical monsoon climate. Hot climate, adequate precipitation and forest provide a suitable environment for the growth and reproduction of mosquitoes and also for malaria transmission. With a complex vector community, Anopheles minimus and Anopheles sinensis were identified as the primary and the secondary vector, respectively $[8,9]$. Year-round malaria transmission occurred in most parts of the border areas prior to the elimination. All four of the parasite species (i.e. $P$. falciparum, P. vivax, P. malariae and P. ovale) detected in the area [10]. There were no natural and artificial barriers along the international border during the period of this study. Thirteen indigenous ethnic minorities live across the international border. The border area of Yunnan is an underdeveloped area with poorer communities, marginalized populations and weaker health services. Civil unrest, unpermitted border crossers, high malaria burden coexist in the border areas of the three neighbouring countries, especially Myanmar. Each of these factors challenged feasibility of border malaria elimination along the international border in Yunnan.

\section{Data sources and collection}

To collect data of malaria cases, intervention activities and control strategies, all available paper-based records related to border malaria surveillance and interventions from 2003 to 2020 were reviewed at Yunnan Institute of Parasitic Diseases (YIPD). Chinese Information System for Disease Control and Prevention (CISDCP) began to cover all Yunnan's counties from 2008 [11, 12], thus, the data of malaria cases and interventions from 2008-2020 were obtained from the CISDCP. In addition, all available documents and literatures about the border malaria situation and control activities in Yunnan and neighbouring countries (Vietnam, Laos and Myanmar) were also reviewed. These literatures and documents include original work records, books, annals, guidelines and operational manuals about malaria control and eliminations.

\section{Data analysis}


First, key actions of border malaria control and elimination were summarized into a list. Second, the strategies and interventions including stratification of malaria areas, treatment of malaria cases, vector control, surveillance and focus response at each stage from malaria hyperendemicty to elimination were described. Third, to present impact of these strategies and interventions, annual parasite incidence (API) of every border county and the overall border area was calculated for selected year 2003, 2006, 2010 and 2013 , respectively. When local transmission was interrupted, malaria is mainly imported from endemic areas of other countries, and calculation of API was not appropriate after 2013 [13], only number of imported malaria cases detected and their infection sources were counted. The years of local malaria free certification by Yunnan Province for eight border prefectures were used to evaluate the impact for elimination interventions. At last, the experiences were used to develop the " $3+1$ " strategy to prevent reintroduction of malaria transmission along the international border in Yunnan [14].

\section{Results}

\section{Key actions}

Table 1 shows the key actions that have significant impact on border malaria control and elimination in Yunnan. 
Table 1

Key actions of malaria control and elimination along international border in Yunnan, China, 2002-2021

\section{Year Actions and results}

2002 An underreported malaria survey among a sample of 30580 people was conducted across Yunnan. Results indicated that mean underreported rate of malaria cases was as high as $88.8 \%$ in Yunnan, and the underreported rate was more than $90 \%$ (range $90.1-97.8 \%$ ) in the border areas [15].

2003 In January, the first round of the China's Global Fund to fight AIDS, Tuberculosis and Malaria (GFATM) malaria program was rolled out in 25 border counties.

2005 On 7th June, the first collaborative document of cross border malaria control was signed between China and Myanmar [17].

2007 In July, the sixth round of the China's GFATM malaria program on cross border malaria control was launched in China's 12 border counties and Myanmar's four special regions [19].

2010 On 1st July, the national malaria elimination action was launched across China including Yunnan's 25 border counties [21].

2012 On 1st January, the tenth round of the China's GFATM malaria program on cross border malaria control was rolled out in China's seven border counties and Myanmar's five special regions [19].

2013- The tenth round of the China's GFATM malaria program on cross border malaria control 2014 stopped in China on 31st December 2013. The second phase of the project was consolidated into the Myanmar's GFATM project from 1st January, 2014 [19].

2014- There was a slight resurgence of malaria incidence in Myanmar's Kachin Special Region II 2016 (KR2) and Shan Special Region II (Wa State) which led to the increase of imported cases of malaria into Yunnan $[27,28]$.

2014 China and Myanmar collaboratively controlled the outbreak of Plasmodium falciparum in Wa State and prevented malaria importation into China [28].

2014 The impact evaluation of cross China-Myanmar border malaria control program during 2007-2013 was carried out. Results indicated that the malaria burden was reduced by $95 \%$ in China's 19 border counties based on the API and by $90 \%$ in Myanmar's five special regions based on the malaria parasite prevalence [19].

2016 The last indigenous malaria case of China was reported from Yingjiang county on ChinaMyanmar border, on 17 April 2016 [23].

2017- China and Myanmar collaboratively controlled the resurgence of malaria incidence in the 2019 malaria hot spot of Laiza and nearby areas, KR2, Myanmar. The number of malaria cases was reduced from 2080 cases in 2016 to 274 cases in 2019 in Laiza and nearby area.

2018 In March, the Yunnan health and Family Planning Commission released "The notification on further standardizing malaria elimination work and process" to clear the responsibility of general health service in malaria surveillance.

2019 In January, the " $3+1$ " strategy for border malaria elimination and preventing reintroduction of malaria transmission was developed and formulated.

2020 In January, Yunnan passed the national technical assessment of malaria elimination. In June, Yunnan passed the finally national assessment of malaria elimination. 


\begin{tabular}{|cl|}
\hline Year & Actions and results \\
\hline 2021 & $\begin{array}{l}\text { In May, The WHO Malaria Elimination Certification Panel conducted field assessment in } \\
\text { Yunnan and visited two border counties, Menglian and Yingjiang, for national malaria } \\
\text { elimination certification. }\end{array}$ \\
\hline $2005-$ & $\begin{array}{l}\text { "The joint malaria control project along China-Myanmar Border" regularly exchanges } \\
\text { information and conducts some activities of malaria control since 2005 [23]. }\end{array}$ \\
\hline
\end{tabular}

\section{Control, 2003-2013}

In 2003, the first year of this case study, a total of 15431 confirmed malaria cases were reported across Yunnan, in which 10349 (67.1\%), mean API of 17.10 per 10000 person-years were reported in the 25 border counties (Additional file 1). A survey of underreported malaria cases reported that the mean underreported rate was $88.8 \%$ across Yunnan, and $90.0 \%$ in the border areas in 2002 (Table 1) [15]. Based on the survey of underreported malaria cases, it was estimated that there were about 100 thousand malaria cases in the border area in 2003. Facing hyperendemicity, approach of "one village, one strategy" that was developed and started in Yunnan in early 1990s was continuously carried out during phase 2003-2013. This strategy categorized all natural villages into four types each year based on malaria incidence in last three years. The definitions and interventions for each type of natural village were listed in Table 2 [16]. To solve problems of high morbidity, specificity and complexity, the strategy of natural village-based stratification and interventions was still conducted in the border county with high malaria burden from 2010 to 2013. Meanwhile, the county-based stratification and interventions documented in "Action plan of China malaria elimination (2010-2020)" were also used for malaria detection and response. During this phase, the API was successfully reduced to 13.54 per 10000 personyears in 2006, followed by 2.26 per 10000 person-years in 2010 and then 0.62 per 10000 person-years in 2013 (Additional file 1). The significant reduction of malaria burden made possible to fully orientate malaria programme from control to elimination in Yunnan. 
Table 2

Malaria area stratification and interventions in border areas, Yunnan, 2003-2013

Tier definition Interventions

Type A village $\mathrm{API} \geq 1 \%$, or malaria clinical attack 1 rate $\geq 10 \%$ last year
1) Mass drug administration for radical cure and preventive treatment;

2) Presumptive treatment of all febrile patients for malaria;

3) Two rounds of indoor residual spraying (IRS) or dipping bed nets with pyrethroid insecticides.

Type A village $\mathrm{API}<1 \%$, or malaria clinical attack 2 rate $<10 \%$ last year, but there are indigenous cases in last 3 years.

1) Radical cure treatment of people with malaria attack history in last 2 years;
2) Presumptive treatment of suspected malaria cases and febrile patients without clear causes for malaria

3) One rounds of IRS or dipping bed nets with pyrethroid insecticides.

Type A village without indigenous cases, only 3 imported cases in last 3 years

Type A village without any malaria cases in last 3 4 years
1) Radical cure treatment of people with malaria attack history in last 2 years;

2) Presumptive treatment of suspected malaria cases and febrile patients without clear causes for malaria;

3) IRS with pyrethroid insecticides to malaria patient's and neighbouring houses

\section{Cross border collaboration between China and Myanmar, 2005-2013}

China and Myanmar signed "The agreement of cross border malaria control" on 7th June 2005 [17]. Supporting from the International Collaboration Department of National Health Commission (the former Ministry of Health) of China, Yunnan has carried out "The joint malaria control project along ChinaMyanmar Border" since 2005 [18]. Due to the limited investment, this project can only focus on personnel training and communication. Under the agreement framework, YIPD and Health Poverty Action (HPA, the former Health Unlimited) that is a UK-based nongovernment organization successfully applied for and implemented the sixth and tenth round of GFATM Malaria projects along China-Myanmar border from 2007 to 2013. The two GFATM projects obtained a total budget of US\$32,512,550. China allocated a total of US\$17,196,071 (52.9\%) to the HPA for control activities in the border areas of Myanmar. When cars, drugs, bed nets and other equipment were included, more than $70 \%$ of the two grants received by China centre for disease control and prevention (CDC) were used in Myanmar. The two GFATM projects distributed a total of 52257 long-lasting insecticidal bed nets, performed 1101979 parasite-based tests (RDTs or microscopy) for malaria, and administered a total of 472356 treatment courses including 
277329 courses for preventive treatments and 195027 courses for clinical treatments. Health education activities were meanwhile conducted to promote these interventions for malaria vector and parasite control. These interventions successfully reduced the malaria burden by $90 \%$ in five special regions of Myanmar, decreasing malaria parasite prevalence from $13.6 \%(95 \% \mathrm{Cl}, 12.7-14.6 \%)$ in March, 2008 , to 1.5\% (95\% Cl, $1.2-2.0 \%)$ in November, 2013. The hyperendemic areas in Myanmar's border areas were reduced to only three main malaria hot spots by 2013, namely, Laiza and nearby areas in Kachin Special Region II (KR2), Salween River Valley in Shan Special Region II and Small Golden Triangle in Shan Special Region IV. The malaria burden was reduced by $95 \%$ in 19 China's counties bordering with Myanamr, decreasing the API from 19.6 per 10000 person-years in 2006 to 0.9 per 10000 person-years in 2013. The reduced malaria burden along China-Myanmar border significantly contributed to the success of malaria elimination in China. For example, only 518 imported malaria cases were detected in Yunnan in 2013 [14, 19].

\section{Elimination, 2014-2016}

Malaria elimination is a set of labor-intensive interventions, which requires a universal coverage of malaria case surveillance and rapid response to malaria foci $[13,14]$. Chinese "1-3-7" strategy requests reporting of malaria cases within one day, confirmation and investigation of malaria cases within three days, and an appropriate public health response to prevent further transmission within seven days [20]. The WHO recommends that elimination phase starts in areas where the first programme reorientation has been achieved, and where health facility data show a malaria incidence of less than 1 infection per 1000 people at risk per year, equal to less than 100 new cases per year in a district with a population of 100 000 people [13]. In China, the smallest unit for elimination is a county, most of counties with a population of over one million. The national malaria elimination program therefore recommended that elimination phase started after API < 1 per 10000 person-years. The action plan of China malaria elimination 20102020 requested to reduce API to less than 1 per 10000 person-years in each border county of Yunnan by the end of 2015. This goal was actually achieved by 2013 with a mean API of 0.62 per 10000 personyears across 25 border counties except Tengchong with API 2.0 per 10000 person-years due to imported malaria cases being calculated into the API and imported cases accounting for more than $95 \%$ of the total cases in Tengchong (Additional file 1). The WHO guideline of malaria elimination does not recommend involvement of imported malaria cases in calculation of API [13]. In Yunnan, malaria elimination action was fully launched in 25 border counties since 2014 .

Yunnan's 104 inland counties kept the pace with country to start malaria elimination action since 2010. The national standards of county stratification for malaria elimination categorized all counties into four tiers: type 1 was presence of confirmed local case(s) in the last three years, with at least one year having an annual incidence $>=1 / 10,000$; type II was presence of confirmed local case(s) in the last three years, annual incidence <1/10,000; type III was no local case for at least three years, only imported cases; and type IV was no history of any local cases, only imported cases [21]. Yunnan categorized its 129 counties into three tiers (no type IV), namely, 19 type I counties with 17 border counties, 55 type II counties with eight border counties, and 55 type III counties based on the national standards in 2010 . On the basis of 
this stratification, every county took malaria elimination as one of the government work objectives, establishing leadership and technical steering team. Intensive surveillance and rapid response were conducted following "The protocol of Yunnan malaria elimination action plan (2010-2020)" [22]. From 2014 to 2016 , a total of 341336 febrile patents were tested by microscopy for malaria. Individual and focus epidemiology investigations and responses were conducted to all 1189 malaria cases or foci following the "1-3-7" work approaches [20]. Strengthened malaria surveillance ensured to detect any parasite infections timely and rapid response to prevent further transmission [22]. The transmission of $P$. falciparum malaria was successfully interrupted since the last locally infected $P$. falciparum case was reported from Cangyuan County in May 2015, and then the $P$. vivax malaria transmission was finally interrupted since the last locally infected $P$. vivax case was reported from Yingjiang County at 17 April 2016 (Additional file 2) [23].

\section{Preventing reintroduction, 2017-2020}

The WHO malaria elimination certification standard is that the chain of indigenous malaria transmission by Anopheles mosquitoes has been interrupted nationwide for at least the past three consecutive years, and a country must also demonstrate the capacity to prevent reintroduction. However, Yunnan borders three malaria endemic countries. Imported malaria can be caused by both border crossers and parasiteinfected Anopheles mosquitoes which fly over the boundary from endemic areas of neighbouring countries. The threat from Vietnam and Lao PDR is slight. The overall incidence of malaria is low in Vietnam with malaria transmission being interrupted in the north of Vietnam [24]. Malaria control has also made rapid progress towards localised elimination goals in the northern provinces of Laos [25]. Yunnan firstly achieved malaria free for at least three years in Honghe Prefectures with three counties only bordering with Vietnam in 2015 [12], and then Wenshan Prefectures with three counties just bordering with Vietnam in 2016. After interruption of malaria transmission, the national stratification for malaria elimination was not suitable for the actual situation any more. To prevent reintroduction effectively, after carefully considering of malaria hyperendemicity in Myanmar and the specificity of 25 border counties, Yunnan further categorized 25 border counties into three tiers in 2017, namely 12 type A counties, seven type $B$ counties, and six type $C$ counties. Type $C$ counties only border with Vietnam, they do not need additional investment for special interventions. The 12 type A counties need more input of human and financial resources to carry out more intensive interventions. Based on results of 291 Anopheles mosquito mark-release-recapture (MMRR) experiments in 143 localities around the world estimated that the mean distance travelled (MDT) of female Anopheles was not more than $2.5 \mathrm{~km}$ [26], assessment of the receptivity and vulnerability for each border community in China and the malaria endemicity within $2.5 \mathrm{~km}$-wide perimeter border areas of Myanmar along the international border, 16 natural villages were proposed to be in the threat of border-spill malaria that was defined as imported malaria caused by parasite-infected Anopheles from border endemic areas of neighbouring countries in 2018 (Additional file 3). Integrated interventions that include proactive and passive detections for the malaria parasites, enhancement and optimization of vector surveillance, further strengthening of timely detection with high-quality confirmed diagnosis and prompt action based on the surveillance results were carried out in these 16 natural villages [14]. The intensive interventions prevented reintroduction 
effectively to make sure the national and the WHO malaria free certification in time. Table 3 shows the years of malaria transmission interrupted and malaria free certificated by Yunnan itself for six border prefectures following the national standards of malaria elimination assessment. The China National Health Commission finally assessed and certificated Yunnan malaria free in June 2020. The WHO Malaria Elimination Certification Panel (MECP) visited two border counties, Menglian and Yingjiang, to conduct field assessment for China's national malaria elimination certification in May 2021. The WHO MECP highly appreciated the infrastructure and equipment, the competence of staff of the health system and supporting organization, the data management and record system during their visits.

Table 3

Time of malaria free certification for eight border prefectures, Yunnan

\begin{tabular}{|lll|}
\hline Prefecture & Transmission interrupted & Malaria free certificated \\
\hline Bordering with Vietnam only & & \\
\hline Honghe & 2012 & 2015 \\
\hline Wenshan & 2013 & 2016 \\
\hline Bordering with Vietnam, Lao PDR and Myanmar & \\
\hline XishuangBanna & 2014 & 2017 \\
\hline Puer & 2016 & 2018 \\
\hline Bordering with Myanmar only & & \\
\hline Baoshan & 2014 & 2017 \\
\hline Lincang & 2016 & 2018 \\
\hline Nujiang & 2016 & May 2019 \\
\hline Dehong & 2016 & Sep 2019 \\
\hline
\end{tabular}

\section{Cross border collaboration, 2014-2020}

China was listed by the World Bank as a mid-high income country in 2012 and was no longer eligible for grants of the GFATM. The second phase of the tenth round of China's GFATM malaria project was consolidated into the Myanmar's GFATM project from 2014. The policy change of the GFATM made China no enough resource investing for malaria control in Myanmar's border areas. Malaria control strategy in Myanmar is different from that of China. For example, Myanmar's strategy only treats malaria cases with laboratory test positivity, and does not permit treatment for suspected malaria cases with antimalarial drugs; only long lasting insecticidal bed nets (LLINs) can be used for vector control, without indoor spraying with insecticides. A slight malaria resurgence appeared in Myanmar's border areas since 2014 [27, 28], which led to an increase of imported malaria in Yunnan, from 358 in 2013 to 594 cases in 2015. To solve the increasing threat of malaria importation, Yunnan strengthened collaboration of 
malaria control with Myanmar, especially Laiza and nearby areas in the KR2. For example, Yunnan provided necessary antimalarial drugs, insecticides and other supplies to the KR2, and sent a technical team from YIPD and Yingjiang County CDC to station in Laiza providing technical assistance. The collaboration and effective interventions reduced the number of malaria cases reported in Laiza and nearby areas from 1936 cases in 2017 followed by 664 cases in 2018 and then 274 cases in 2019. Decreased malaria burden in the KR2 contributed the reduced number of malaria importation in Yingjiang County, from 179 cases in 2017 followed by 106 cases in 2018, and then 92 cases in 2019 and 75 cases in 2020. The cross border collaboration not only helped achieving malaria free and preventing reintroduction, but also reduced the work load of epidemiological investigations and responses to imported malaria in Yunnan.

\section{Discussion}

The WHO has awarded China a malaria-free certification on 30 June 2021[29]. The WHO certification of malaria elimination requires applicant countries to provide evidence that: 1) local malaria transmission has been fully interrupted, resulting in zero indigenous human malaria cases for at least the past 3 consecutive years (36 months), and 2) an adequate programme for preventing reintroduction of malaria transmission is fully functional throughout the country [13]. Border malaria is one of the most intractable problems that countries face today in their path to malaria elimination. Interruption of malaria transmission and continuous maintenance of malaria free in Yunnan's border areas determined whether the WHO would certificate malaria elimination for the Whole China in time [19]. This case study described experiences and lessons learnt from hyperendemicity to elimination along international border in Yunnan. The WHO recommends that the stratification should be initially done at the lowest geographical level for which operational decisions can be made [13]. In 1990s, Yunnan developed the natural village -based stratification for cost-effective interventions, and the stratification and intervention approaches were adjusted every year [16]. However, the strategy could not fully be carried out because of the lack of resources. Prior to 2003 , local people were facing problems of not enough accessibility to laboratorybased diagnosis, effective treatment and vector control, which led to a high underreported rate [15]. The difficulty led to malaria unable to fully be controlled. Proper investment made possible to fully carry out natural village-based stratification and interventions from 2003 to 2013. The interventions reduced malaria burden dramatically, decreasing overall API from 17.10 per 10000 person-years in 2003 to 13.54 per 10000 person-years in 2006, followed by 2.26 per 10000 person-years in 2010 and 0.62 per 10000 person-years in 2013 (Additional file 1). The overall API was still as high as 13.54 per 10000 person-years in 2006 was mostly due to the reduced underreported rate, and the actual reduction of malaria burden should be much more than the figure.

Accurate stratification of malaria transmission intensity is essential to effectively targeted interventions in Yunnan. The WHO recommends that interventions are expected to change the epidemiology of malaria rapidly and profoundly, the stratification of malaria maps should be revised frequently. As transmission intensity was progressively reduced, stratification needs to include vulnerability and receptivity to malaria [13]. "Action plan of China malaria elimination (2010-2020)" defined a county was a unit of elimination. 
The 25 border counties was categorized into two tiers according to the national stratification standards for malaria elimination, namely 17 type I counties and eight type II counties [21, 22]. Yunnan has interrupted malaria transmission in 2017 , and the national stratification standards for malaria elimination were not appropriate for the actual situation any more. To effectively guide resource allocation and use more targeted control strategy for preventing reintroduction of malaria transmission, Yunnan further stratified the 25 border counties into three types of A, B and C; and also identified 16 natural villages with high risks of border-spill malaria in 2018 (Additional file 3). Based on the experiences of malaria control from hyperendemicity to elimination, the approach of " $3+1$ " strategy was designed to provide: 1 ) comprehensive and intensive malaria interventions along a $2.5 \mathrm{~km}$ wide perimeter with the international border with China to prevent border-spill malaria, 2) community-based malaria surveillance and identify international migrants with malaria cues in the frontier townships, and 3) consolidate surveillance into normal health services to maintain vigilance of health personnel to malaria cues. The +1 emphasizes the need to strengthen collaboration with neighbouring countries to reduce their malaria burden with a clear focus on border areas with China [14]. The " $3+1$ " strategy is in accordance with the principle of the WHO recommended malaria elimination strategy [13].

As a technical experience, drug-based treatment is the primary intervention in malaria control and elimination. Asymptomatic and submicroscopic parasite density, especially for Plasmodium vivax, and limitations of microscopist ability and Rapid diagnostic tests may lead to under detection or misdiagnosis. Antimalarial treatment for malaria should not just target confirmed malaria cases [27, 30]. Based on experiences in the border areas, mass drug administration can rapidly reduce malaria burden in hyperendemic areas, however, it might not be necessary for mesoendemic situation. Treatment for all confirmed, clinical and suspected cases combined with vector controls should be considered to try in the mesoendemic areas [27, 30].

\section{Conclusions}

Border malaria elimination is an intensive intervention. Malaria from hyperendemicity to elimination along the international border in Yunnan can be attributed to the local context based strategy including governmental commitment, effective interventions and collaboration with neighbouring countries. Although malaria has been eliminated and reintroduction of malaria transmission prevented, malaria importation from endemic areas of neighbouring countries is still a continuous threat in the border areas. The comprehensive interventions are continuously essential in preventing reintroduction of malaria transmission. However, other border areas should do their own intervention trials to develop their own strategy for malaria control and elimination.

\section{Abbreviations}

API: Annual Parasite Incidence; WHO: World Health Organization; GMS: Greater Mekong Subregion; YIPD: Yunnan Institute of Parasitic Diseases; CISDCP: Chinese Information System for Disease Control and Prevention; GFATM: Global Fund to fight AIDS, Tuberculosis and Malaria; CDC: Centre For Disease Control 
And Prevention; RDT: Rapid Diagnostic Diseases; KR2: Kachin Special Region Il; MDT: Mean Distance Travelled; MECP: Malaria Elimination Certification Panel; LLN: Long Lasting Insecticidal Bed Nets.

\section{Declarations}

\section{Acknowledgements}

Not applicable

\section{Authors' contributions}

HNZ, JWX and HL conceived the work. JWX and HL reviewed literatures and wrote the manuscript. YWZ, YD, ZRL, CLZ, QYC, CW, KXD and PT provided critical comments. All authors read and approved the final manuscript.

\section{Funding}

This work was supported by the National Natural Science Foundation of China (No. 81560543 and 81673113).

\section{Availability of data and materials}

Not applicable.

\section{Ethics approval and consent to participate}

Not applicable.

\section{Consent for publication}

Not applicable.

\section{Competing interests}

The authors declare that they have no competing interests.

\section{References}

1. WHO. World Malaria Report 2019. Geneva: World Health Organization, 2019. Accessed 3 Mar 2020.

2. WHO. World Malaria Report 2020. Geneva: World Health Organization, 2020. Accessed 6 Feb 2021.

3. WHO. Global technical strategy for malaria 2016-2030. Geneva: World Health Organization; 2015. Accessed 27 Feb 2016.

4. WHO. The E-2020 initiative of 21 malaria eliminating countries-2019 progress report. Geneva: World Health Organization; 2019. 
5. WHO. Strategy for malaria elimination in the Greater Mekong Subregion: 2015- 2030. Geneva: World Health Organization; 2015. Accessed 27 Feb 2016.

6. Lai S, Sun J, Ruktanonchai N, Zhou S, Yu J, Routledge I, et al. Changing epidemiology and challenges of malaria in China towards elimination. Malar J 2019; 18:107.

7. Wang D, Li S, Cheng Z, Xiao N, Cotter C, Hwang J, et al. Transmission risk from imported Plasmodium vivax malaria in the China-Myanmar Border Region. Emerging Infectious Diseases 2015; 21 (10): 1861-1864.

8. Hii J, Rueda L. Malaria vectors in the Greater Mekong Subregion: Overview of malaria vectors and remaining challenges. Southeast Asian J Trop Med Public Health. 2013; 44 (Suppl 1): 73-165, discussion 306-7.

9. Dong XS. The malaria vectors and their ecology in Yunnan Province (in Chinese). Chin J Parasit Dis. 2000;13: 144-7.

10. Yang HL and Zhou HN. Yunnan malaria. Kunming: Yunnan Science and Technology Press (in Chinese), 2015.

11. Xu JW, Li Y, Yang HL, Zhang J, Zhang ZX, Yang YM, et al. Malaria control along China-Myanmar Border during 2007-2013: an integrated impact evaluation. Inf Dis Poverty. 2016; 5:75

12. Xu JW, Li JJ, Guo HP, Pu SW, Li SM, Wang RH, et al. Malaria from hyperendemicity to elimination in Hekou County on China-Vietnam border: an ecological study. Malar J. 2017; 16: 66.

13. WHO. Malaria elimination: a field manual for low and moderate endemic countries. Geneva: World Health Organization; 2014. Accessed 20 Feb 2015.

14. Xu JW, Lee R, Lin ZR, Zhou YW, Shen HM, Zhou HN, et al. Intensive Surveillance, Rapid Response and Border Collaboration for Malaria Elimination: China Yunnan's "3+1" Strategy. Malar J. 2021; 20, 396. https://doi.org/10.1186/s12936-021-03931-8.

15. Zhang ZX, Zhou S, Xu JW, Du ZW, Chen GW, Li L, Zhou HN, Guo XF, Wu C, Li JS, Liu H, Xu SY, Wang LB, Chen ZW, Yang YC, Xia M, Li RJ. Investiagtion on missing-report of malaria cases in Yunnan, China. Chinese Journal of Parasitic Disease Control. 2004; 17 (1): 25-27 (in Chinese).

16. Xu JW, Wang WR, Xu SY. Malaria situation and control in border areas and Yuanjiang River Basin of Yunnan Province in 1995. Chinese Journal of Parasitic Disease Control 1998; 11(1): 71 (in Chinese).

17. China $\mathrm{MOH}, \mathrm{Myanmar} \mathrm{MOHS}$. The agreement of malaria control in China-Myanmar border areas. Kunming: China ministry of health, Ministry of Health and Sports of Myanmar; 2005. Accessed 9 Jun 2005.

18. Zhou H, Du L, Yang H, Zhang Z. Innovative decade of cross-border joint prevention \& control project of malaria and dengue fever in Yunnan China-GMS areas. Kunming: Yunnan science and technology press, 2015.

19. Xu JW, Li Y, Yang HL, Zhang J, Zhang ZX, Yang YM, et al. Malaria control along China-Myanmar Border during 2007-2013: an integrated impact evaluation. Inf Dis Poverty. 2016; 5:75. 
20. Cao J, Sturrock HJW, Cotter C, Zhou S, Zhou H, et al. Communicating and Monitoring Surveillance and Response Activities for Malaria Elimination: China's "1-3-7" Strategy. PLoS Med. 2014; 11(5): e1001642. doi:10.1371/journal.pmed.1001642.

21. Ministry of Health of the People's Republic of China. Action plan of China malaria elimination (20102020). 2012. Available: http://www.gov.cn/gzdt/ att/att/site1/20100526/001e3741a2cc0d67233801. doc. Accessed 15 October 2013.

22. YHD. The protocol of Yunnan malaria elimination action plan (2010-2020) (in Chinese). Kunming: Yunnan health department; 2010. Accessed 27 Feb 2019.

23. Zhao X, Sun $X$, Yang $H$, Zhou D, Yang J, Guo T, et al. A report of the last indigenous malaria case in Yunnan (in Chinese). China Trop Med. 2020; 20: 325-328.

24. Thanh PV, Hong NV, Van NV, Malderen CV, Obsomer V, Urgell AR, et al. Epidemiology of forest malaria in Central Vietnam: the hidden parasite reservoir. Malaria Journal. 2015; 14:86.

25. Kounnavong S, Gopinath D , Hongvanthong B, Khamkong C, Sichanthongthip O. Malaria elimination in Lao PDR: the challenges associated with population mobility. Infectious Diseases of Poverty. 2017; 6:81.

26. Guerra AC, Reiner JRR, Perkins TA, Lindsay SW, Midega JT, Brady O, et al. A global assembly of adult female mosquito mark-release-recapture data to inform the control of mosquito-borne pathogens. Parasites \& Vectors. 2014; 7:276.

27. Liu H, Xu J-W, Bi Y. Malaria burden and treatment targets in Kachin Special Region II, Myanmar from 2008 to 2016: A retrospective analysis. PLoS one. 2018; 13 (4): e0195032. https://doi.org/10.1371/journal.pone.0195032.

28. Liu H, Xu JW, Yang HL, Li Mei, Sun CD, Yin YJ, et al. Investigation and control of a Plasmodium falciparum malaria outbreak in Shan Special Region II of Myanmar along the China-Myanmar Border from June to December 2014. Inf Dis Poverty. 2016; 5:32.

29. Burki T. Triumph in China as it is certified malaria-free by WHO. Lancet Inf Dis. 2021;21:1220-1.

30. Xu JW, Lee R, Li XH, Liu Hui. Transition of radical, preventive and presumptive treatment regimens for malaria in China: a systematic review. Malar J. 2021; 20:10 https://doi.org/10.1186/s12936-02003535-8.

\section{Supplementary Files}

This is a list of supplementary files associated with this preprint. Click to download.

- IDPR1additionalfile22Sep2021.docx 